\title{
Modification of isotropic coal-tar pitch by acid treatments for carbon fiber melt-spinning
}

\author{
Mi Jung Yoo ${ }^{1}$, Hyo Jun Ko ${ }^{2}$, Yun-Soo Lim ${ }^{2}$ and Myung-Soo Kim ${ }^{1, \$}$ \\ ${ }^{1}$ Department of Chemical Engineering, Myongji University, Yongin 449-728, Korea \\ ${ }^{2}$ Department of Materials Science and Engineering, Myongji University, Yongin 449-728, Korea
}

\section{Article Info}

Received 10 July 2014

Accepted 23 September 2014

*Corresponding Author

E-mail: myungkim@mju.ac.kr Tel: +82-31-330-6391

\section{Open Access}

DOI: http://dx.doi.org/

10.5714/CL.2014.15.4.247

This is an Open Access article distributed under the terms of the Creative Commons Attribution Non-Commercial License (http://creativecommons.org/licenses/ by-nc/3.0/) which permits unrestricted non-commercial use, distribution, and reproduction in any medium, provided the original work is properly cited.

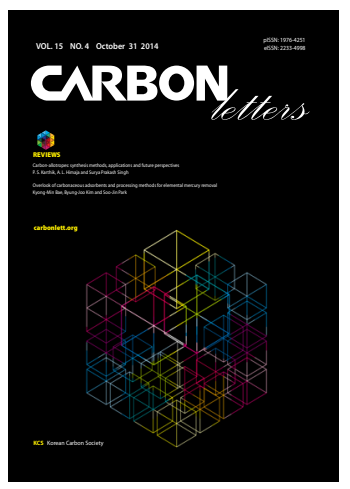

http://carbonlett.org

pISSN: $1976-4251$

eISSN: 2233-4998

Copyright $\odot$ Korean Carbon Society

\begin{abstract}
In this work, thermal treatment accompanied with different acid treatments was applied to a commercial coal tar pitch (CTP) to obtain a spinnable precursor pitch for carbon fiber. In the case of thermal treatment only, a relatively high reaction temperature of between $380^{\circ} \mathrm{C}$ and $400^{\circ} \mathrm{C}$ was required to obtain a softening point (SP) range of $220^{\circ} \mathrm{C}-260^{\circ} \mathrm{C}$ and many meso-phase particles were created during the application of high reaction temperature. When nitric acid or sulfuric acid treatment was conducted before the thermal treatment, the precursor pitch with a proper SP range could be obtained at reaction temperatures of $280^{\circ} \mathrm{C}$ $300^{\circ} \mathrm{C}$, which were about $100^{\circ} \mathrm{C}$ lower than those for the case of thermal treatment only. With the acid treatments, the yield and SP of the precursor pitch increased dramatically and the formation of meso-phase was suppressed due to the lower reaction temperatures. Since the precursor pitches with acid and thermal treatment were not spinnable due to the inhomogeneity of properties such as molecular weight distribution and viscosity, the CTP was mixed with ethanol before the consecutive nitric acid and thermal treatments. The precursor pitches with ethanol, nitric acid, and thermal treatments were easily spinnable, and their spinning and carbon fiber properties were compared to those of air blowing and thermal treated CTP.
\end{abstract}

Key words: coal tar pitch, acid treatment, carbon fiber, melt-spinning

\section{Introduction}

Coal tar pitch (CTP) is a byproduct of the coking process and isotropic CTP is cheaper than other carbon fiber precursors such as polyacrylonitrile (PAN). Since CTP consists of a variety of different phenols and polycyclic aromatic hydrocarbons $[1,2]$, it must be properly treated to obtain spinnable pitches. In order to achieve lower price and high performance pitch carbon fiber, CTP has been restructured by purifying processes $[3,4]$, thermal and acid treatments [5], air blowing [6-9], thermal treatment [10], and modification with a polymer [11-13]. These treatments affect the properties of the carbon fiber precursor because they cause a considerable change in the chemical composition of pitches. As a result, the softening point (SP) and yield of the precursor pitch can be increased and the mechanical properties of the resultant carbon fiber can be improved. To prepare an isotropic spinnable pitch, CTP should meet two important qualifications: it must have a high SP and should maintain isotropic characteristics or be transformed to a $100 \%$ meso-phase (anisotropic) pitch. To obtain excellent spinnability, CTP should have homogeneity in properties such as a short range of molecular weight distribution and viscosity. However, with treatment at high temperatures, isotropic CTP usually generates mesophase particles. To control the generation of a meso-phase, various methods have been used such as acid treatments to reduce reaction temperatures [5], and adding polymers to reduce or increase meso-phase formation [11-13]. Isotropic CTP is less costly than meso-phase CTP or PAN because of its low temperature manufacturing conditions. It is also suitable 
for spinning processes because of its low viscosity. However, since it has no directional characteristic, its carbon fiber has poor mechanical properties such as tensile strength and tensile modulus. The isotropic pitch fiber can be used in commonplace applications such as sporting goods and insulators [14]. Most previous studies on CTP have the limitation that modified CTPs were only analyzed but not applied to products such as carbon fiber. The goal of this study is to prepare a spinnable precursor pitch with proper properties for carbon fiber using isotropic CTP. To accomplish this goal, we investigated various treatments on CTP such as thermal treatment only and sulfuric acid treatment or nitric acid treatment before thermal treatment. The properties of the modified CTP and its fiber were analyzed by an elemental analysis, insoluble weight portion, thermogravimetric analysis (TGA), Fourier transform infrared spectroscopy (FT-IR), polarization microscope analysis, and scanning electron microscopy (SEM). Through these procedures, the optimal reaction conditions to prepare the spinnable precursor pitch at low production cost were demonstrated. In particular, this study focused on obtaining a high yield of precursor pitch with high SP and homogeneity for a continuous melt-spinning process.

\section{Experimental}

\subsection{Raw pitch}

A commercial CTP provided by OCI Co. Ltd. (Korea) was used as the raw material. Its quinoline insoluble (QI) portion was eliminated by a centrifugal separator. The detailed information of CTP is presented in Table 1.

\subsection{Pitch modification}

\subsubsection{Thermal treatment}

Thermal treated CTP is denoted as CTP-T. Thermal treatment of CTP was performed to compare the properties with those of acid treatments. $100 \mathrm{~g}$ of pitch was placed in a $500 \mathrm{~mL}$ round flask and heated from room temperature to $380^{\circ} \mathrm{C}-400^{\circ} \mathrm{C}$ at a heating rate of $5^{\circ} \mathrm{C} / \mathrm{min}$, and maintained with continuous stirring for $2 \mathrm{~h}$. A vacuum pump with a cold trap was connected to the flask to eliminate emission gas.

\section{Table 1. Properties of raw coal tar pitch}

\section{Properties}

$\begin{array}{cc}\text { Softening point (ring \&ball) } & 28 \\ \text { Specific gravity } & 1.236 \\ \text { Viscosity (at 80) } & 271 \mathrm{cps} \\ \text { Viscosity (at 110) } & 60 \mathrm{cps} \\ \text { Quinoline insoluble } & <0.1 \% \\ \text { Toluene insoluble } & 7.7 \% \\ \text { Cocking value } & 27.2 \% \\ \text { Ash } & <0.01 \%\end{array}$

\subsubsection{Acid and thermal treatments}

Sulfuric acid and nitric acid treated CTP are denoted as CTP$\mathrm{S}$ and CTP-N, respectively. The amounts of acid were determined such that the addition of $11 \mathrm{~g}$ of $95 \%$ sulfuric acid or 14 $\mathrm{g}$ of $60 \%$ nitric acid to $100 \mathrm{~g}$ of CTP was suitable to obtain a proper SP of the precursor pitch. Each acid reagent was injected to the melted CTP at $70^{\circ} \mathrm{C}$. After mixing CTP with acid by stirring for $30 \mathrm{~min}$, the mixture was heated to $280^{\circ} \mathrm{C}-300^{\circ} \mathrm{C}$ at a heating rate of $5^{\circ} \mathrm{C} / \mathrm{min}$ and maintained with continuous stirring and applied vacuum for $2 \mathrm{~h}$.

2.2.3. Nitric acid treatment with ethanol and thermal treatment

In order to improve the homogeneity, the CTP was mixed with ethanol before the consecutive nitric acid and thermal treatments. Nitric acid treated CTP with ethanol is denoted as CTP-NE. Since ethanol is an amphiphilic solvent, it may help obtain a more homogenous mixture of CTP and nitric acid. Fifty mililiters of ethanol was premixed with $100 \mathrm{~g}$ of pitch at $70^{\circ} \mathrm{C}$. Then $14 \mathrm{~g}$ of $60 \%$ nitric acid was injected to the mixture and the following steps were the same as in the previous acid treatments.

\subsection{Preparation of carbon fibers}

Two precursor pitches (CTP-NE: SP236 and CTP-AB: SP284) were used for fiber spinning. The CTP-AB (CTP modified with air blowing and thermal treatments, SP $284^{\circ} \mathrm{C}$, TI $65.8 \%$, QI 21.4\%) was provided from OCI Co. Ltd. and was also spun to compare the spinning and fiber properties with those of CTP-NE. The modification procedure of CTPNE: SP236 (nitric treated pitch with ethanol, SP $236^{\circ} \mathrm{C}$ ) is represented in Fig. 1. Modified CTPs were spun into green fibers through a single-hole spinneret $($ diameter $=0.3 \mathrm{~mm}, \mathrm{~L} / \mathrm{D}$ $=2$ ) by pressurized nitrogen gas at a spinning temperature of $30^{\circ} \mathrm{C}-40^{\circ} \mathrm{C}$ above the SP and a winding speed of $500 \mathrm{~m} / \mathrm{min}$. The stabilization and carbonization processes were conducted using an electric tube furnace. Stabilization temperature was $10^{\circ} \mathrm{C}$ higher than the SP of the precursor pitch for $10 \mathrm{~h}$ under an air flow. In the carbonization process, the stabilized fibers were heated to $1000^{\circ} \mathrm{C}$ at a heating rate of $2.5^{\circ} \mathrm{C} / \mathrm{min}$ and maintained for $3 \mathrm{~min}$ in a nitrogen atmosphere.

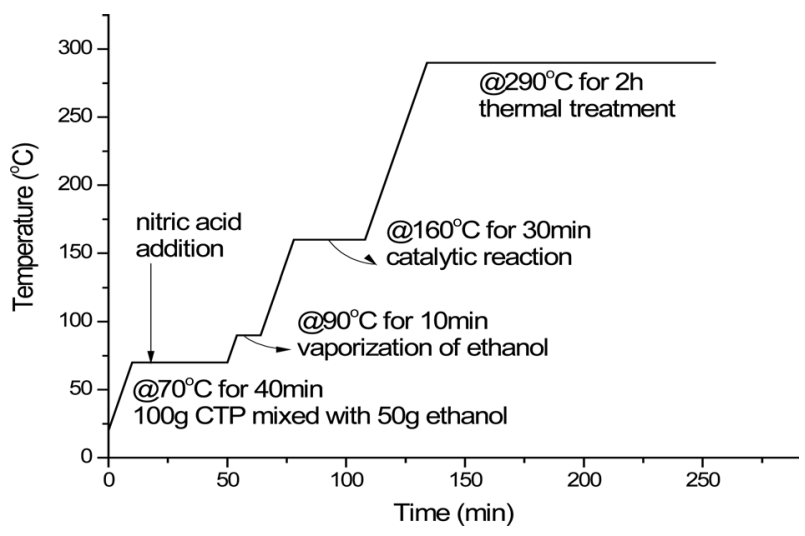

Fig. 1. Modification procedure of coal tar pitch (CTP)-NE: SP236. 


\subsection{Characterization of precursor pitches and carbon fibers}

Contents of carbon, hydrogen, and nitrogen for various pitch samples were measured by an elemental analyzer (EA1112; Thermo Fisher Scientific Co.). The furnace temperature was $900^{\circ} \mathrm{C}$ and the oven temperature was $500^{\circ} \mathrm{C}$. Helium and oxygen flow rates were $140 \mathrm{~mL} / \mathrm{min}$ and $250 \mathrm{~mL} / \mathrm{min}$, respectively. SP of the modified CTPs was measured by the Mettler method (KSM1808) using a dropping point system (DP70, Mettler Toledo Co.). The diameter of the nozzle is $6.35 \mathrm{~mm}$. The starting temperature was about $20^{\circ} \mathrm{C}$ lower than the SP of the sample and the heating rate was $2^{\circ} \mathrm{C} / \mathrm{min}$. Two measurements were conducted for each sample and their average was taken. A soxhlet extractor was used to measure insoluble mass portions of the modified CTPs. One gram of dried pitch sample was pulverized ( $\sim 100 \mathrm{mesh})$ and located in a dried cellulose thimble filter (diameter $28 \mathrm{~mm}$, height $100 \mathrm{~mm}$ ). The pitch samples were extracted for $2 \mathrm{~h}$ by hexane and toluene. After extraction, the thimbles with remaining insolubles were situated in a dry oven and the solvents were evaporated for $24 \mathrm{~h}$ at $70^{\circ} \mathrm{C}$ before the weight measurement. QI could not be measured by the Soxhlet extractor because of quinoline's high boiling point. The pitch sample was dissolved in warm quilonine several times and the solution with QI was filtered using an aspirator. It was then washed by acetone and the weight of residue was measured. FT-IR spectra of the modified CTPs were obtained using a Fourier transform infrared spectrophotometer (DRS-800, Shimazu Co.). The spectra were obtained in a range of $400-4000 \mathrm{~cm}^{-1}$. A thermogravimetric analyzer (SDT 851, Mettler Toledo Co.) was used to demonstrate the carbon yield of CTP to carbon fiber and the approximate distribution of molecular weight. The modified CTPs were heated from room temperature to $1000^{\circ} \mathrm{C}$ at a heating rate of $10^{\circ} \mathrm{C} / \mathrm{min}$ in a nitrogen atmosphere. The viscosity of modified CTPs depending on temperature was measured using a stress controlled rheometer (MCR 300, Anton Paar Co.). The shear rate was $10 \mathrm{~s}^{-1}$ and the viscosity was measured with increasing temperature from the SP of samples in a nitrogen atmosphere. A SEM (S-3500N Hitachi Co.) was employed to analyze the diameter of carbon fibers with platinum coating. Optical microscopy images of the modified CTPs that were mounted with epoxy resin and polished were observed on a polarized light microscope (DM 2700P, Leica Co.)

\section{Results and Discussion}

\subsection{Characterization of raw and modified pitches}

3.1.1. Yield, SP, and elemental analysis

The yield, SP, and elemental content of various CTPs depending on the reaction temperature are shown in Table 2 . When the raw CTP was only thermally treated, a relatively high temperature of between $380^{\circ} \mathrm{C}$ and $400^{\circ} \mathrm{C}$ was required to prepare a precursor pitch with a proper $\mathrm{SP}$ of $220^{\circ} \mathrm{C}-260^{\circ} \mathrm{C}$. As higher temperature was applied, a larger amount of gas was produced and eliminated by the vacuum pump, resulting in an increased SP and decreased yield. The precursor pitch should have a high SP at least $180^{\circ} \mathrm{C}$ because the combination reaction between oxygen and pitch starts above $180^{\circ} \mathrm{C}$ on stabilization process. With increasing SP, higher stabilization temperature and shorter stabilization time can be used. As a result, the production cost of carbon fiber can be reduced because the stabilization step is the most expensive process. However, high $\mathrm{SP}$ over $300^{\circ} \mathrm{C}$ is not desirable due to difficulty of spinning. We therefore set a target SP range of $230^{\circ} \mathrm{C}-290^{\circ} \mathrm{C}$. In the case of acid treatments, the precursor pitch with a proper SP range could be obtained at temperatures of $280^{\circ} \mathrm{C}-300^{\circ} \mathrm{C}$, which were about $100^{\circ} \mathrm{C}$ lower than those for the thermal treatment only. Furthermore, the yield and SP of the precursor pitch increased dramatically with the acid treatments. The increased yield and low temperature of thermal treatment are very useful to lower the production cost of carbon products manufactured from the pitch precursors. Nitric acid treatment (CTP-N) showed even 15\%-20\% higher yields than sulfuric acid treatment (CTP-S). However, the SP of CTP$\mathrm{N}$ samples could not be measured by the Metter method because

Table 2. Reaction temperature, yield, and softening point of thermal and acid treated pitches

\begin{tabular}{|c|c|c|c|c|c|c|c|}
\hline \multirow{2}{*}{ Pitch } & \multirow{2}{*}{ Reaction temperature $\left({ }^{\circ} \mathrm{C}\right)$} & \multirow{2}{*}{$\begin{array}{l}\text { Yield } \\
(\%)\end{array}$} & \multirow{2}{*}{$\begin{array}{l}\text { Softening point } \\
\left({ }^{\circ} \mathrm{C}\right)\end{array}$} & \multicolumn{4}{|c|}{ Elemental fraction } \\
\hline & & & & $\mathrm{C}$ & $\mathrm{H}$ & $\mathrm{N}$ & $\mathrm{C} / \mathrm{H}$ \\
\hline \multirow{2}{*}{ СТР-Т } & 380 & 36 & 220 & 94.40 & 3.76 & 0.95 & 2.09 \\
\hline & 400 & 34 & 260 & 94.55 & 3.87 & 1.06 & 2.04 \\
\hline \multirow{2}{*}{ CTP-S } & 280 & 62 & 220 & 90.57 & 4.22 & 1.37 & 1.79 \\
\hline & 300 & 59 & 270 & 91.36 & 4.02 & 1.33 & 1.89 \\
\hline \multirow{2}{*}{ CTP-N } & 280 & 81 & - & 91.49 & 3.87 & 2.31 & 1.97 \\
\hline & 300 & 77 & - & 92.88 & 4.00 & 2.17 & 1.93 \\
\hline \multirow{2}{*}{ CTP-NE } & 280 & 79 & 220 & 92.58 & 4.22 & 1.75 & 1.83 \\
\hline & 300 & 70 & 290 & 92.58 & 4.07 & 1.97 & 1.90 \\
\hline
\end{tabular}

CTP: coal tar pitch. 
Table 3. Insoluble mass portion of thermal and acid treated pitches

\begin{tabular}{|c|c|c|c|c|c|c|}
\hline & Reaction temperature $\left({ }^{\circ} \mathrm{C}\right)$ & Softening point $\left({ }^{\circ} \mathrm{C}\right)$ & HI (\%) & TI $(\%)$ & QI (\%) & $\beta$-resin $(\%)$ \\
\hline \multirow{2}{*}{ СТP-T } & 380 & 220 & 95.2 & 47.9 & 16.2 & 31.7 \\
\hline & 400 & 260 & 96.5 & 72.0 & 27.3 & 44.8 \\
\hline \multirow{2}{*}{ CTP-S } & 280 & 220 & 88.7 & 43.0 & 21.4 & 21.6 \\
\hline & 300 & 270 & 94.9 & 88.7 & 49.8 & 39.0 \\
\hline \multirow{2}{*}{ CTP-N } & 280 & - & 79.9 & 62.1 & 50.1 & 12.1 \\
\hline & 300 & - & 94.4 & 78.3 & 50.8 & 27.5 \\
\hline \multirow{2}{*}{ CTP-NE } & 280 & 220 & 77.9 & 45.3 & 21.2 & 24.1 \\
\hline & 300 & 290 & 91.2 & 72.0 & 23.9 & 48.1 \\
\hline
\end{tabular}

CTP: coal tar pitch, HI: hexane insoluble, TI: toluene insoluble, QI: quinoline insoluble.

of the low fluidity near the SP, indicating that the CTP-N was not spinnable. As the CTP was mixed with ethanol before the consecutive nitric acid and thermal treatments, the SPs of CTP-NE could be measured and were in the same range as those of CTP-S. The elemental analysis showed that the CTP-T has a relatively high $\mathrm{C} / \mathrm{H}$ ratio over 2.0 as compared with sulfuric and nitric acid treated CTPs. It appears that acid treatment increased hydrogen bonds. There was a small increase of nitrogen content in CTP$\mathrm{N}$, indicating the formation of nitrogen functional groups during the polymerization of CTP with nitric acid [5].

\subsubsection{Solubility results}

Insoluble mass portions of the modified CTPs are shown in Table 3. Beta resin refers to the portion of toluene insoluble (TI) minus QI. TI, QI, and beta-resin portions influence the formation of meso-phase. The existence of primary QI in isotropic pitch has an adverse effect on homogeneity with the creation of meso-phase particles and causes a viscosity differential. Isotropic QIs are asphaltene-like molecules scattered in modified CTP prepared by air blowing and acid treatment. Although the isotropic QI increases coke yield compared to untreated pitch, a high QI over 40\% represents low fluidity becoming cokes [1517]. Hexane insoluble (HI) of the thermal treated pitch (CTP-T) was over $95 \%$. This means that the low molecular-weight potion of raw pitch was effectively eliminated by the vacuum or grew to high-molecular weight of polymer by the poly-condensation reaction. TI, QI, and beta resin of CTP-T treated at $400^{\circ} \mathrm{C}$ were much higher than those treated at $380^{\circ} \mathrm{C}$. As higher reaction temperature was applied, a larger amount of low molecular weight portion disappeared by the activated poly-condensation reaction. Insoluble portions of CTP-S were very dependent on the reaction temperature. This means that the sulfuric acid treatment is very difficult to control the optimum reaction temperature. TI and QI of CTP-S treated at $280^{\circ} \mathrm{C}$ were $43.0 \%$ and $21.4 \%$, respectively, indicating that CTP-S had a large portion of low molecular compound. When the CTP-S was treated at $300^{\circ} \mathrm{C}$, which was just $20^{\circ} \mathrm{C}$ higher, TI and QI increased more than twofold and beta resin increased from $21.6 \%$ to $39 \%$. However, since QI was very high at about $50 \%$, it was not suitable for spinning. The CTP-N also had a high QI of about $50 \%$ and beta-resin portions were relatively low. In addition to the pre-

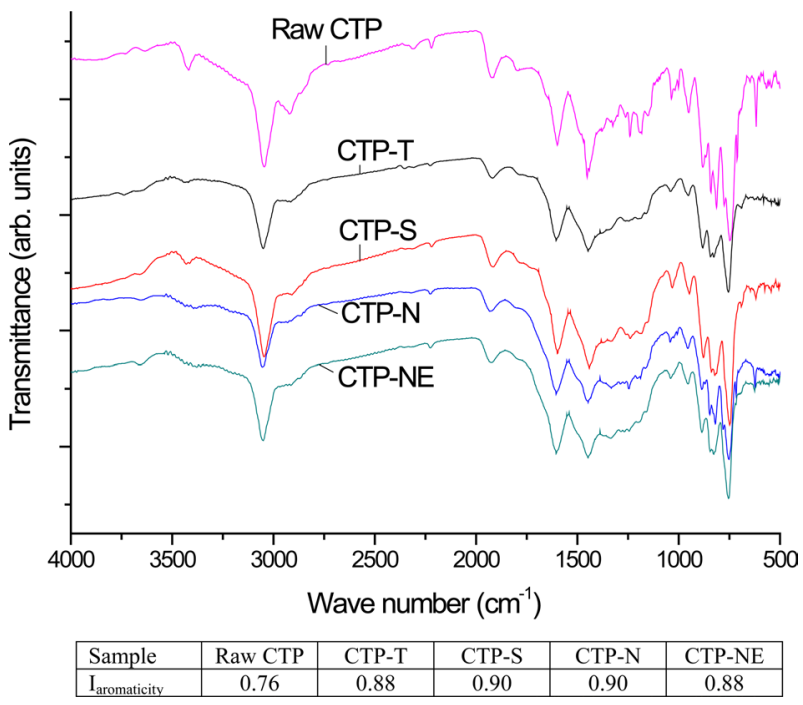

Fig. 2. Fourier transform infrared spectra and aromaticity indexes of various coal tar pitches (CTPs).

vious SP analysis, these insoluble data meant that the CTP-N was not spinnable. To solve this problem, the CTP was mixed with ethanol before the consecutive nitric acid and thermal treatments. The CTP-NE showed slightly reduced TI and about $30 \%$ reduced QI as compared to the CTP-N. As a result, higher portions of beta-resin in a range of $24 \%-48 \%$ could be obtained and the obtained precursor pitch showed excellent spinnability, exhibiting continuous spinning for more than $30 \mathrm{~min}$.

\subsubsection{FT-IR results of modified pitches}

To examine the chemical structure of precursor pitch, their FT-IR spectra are shown in Fig. 2. Compared to the raw CTP, the modified CTPs made noticeable reductions of absorption at $2920 \mathrm{~cm}^{-1}$ but there are no significant differences among the various modified pitches. Because the formation of notable new bonds and the intensity change of existing spectra were not observed with the acid treatments, it was demonstrated that sulfuric and nitric acid helped the poly-condensation at low temperature, playing as the role of a catalyst, without remaining as chemi- 

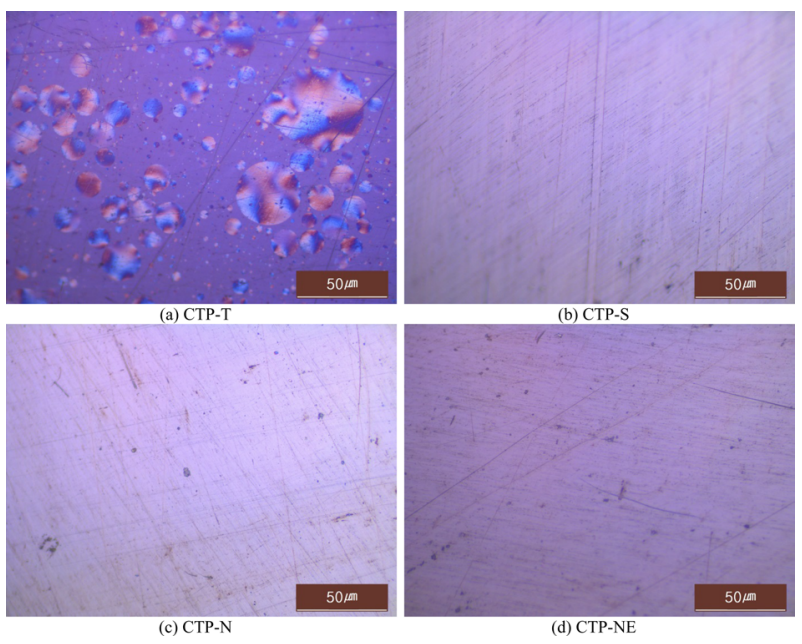

Fig. 3. Optical textures of thermal and acid treated pitches by polarizing microscope. CTP: coal tar pitch.

cal bonds. Some functional groups of the modified CTP were observed as aromatic $(3050,1161,1505,1052,872,811,750$, and $\left.438 \mathrm{~cm}^{-1}\right)$ and aliphatic $\left(2955,2920,2855\right.$, and $\left.1450 \mathrm{~cm}^{-1}\right)$ groups. In particular, we can see the existence of an aromatic $\mathrm{C}-\mathrm{H}$ stretch at $3050 \mathrm{~cm}^{-1}$ and the stretching of aromatic $\mathrm{C}=\mathrm{C}$ at $1611-1470 \mathrm{~cm}^{-1}[18-20]$. The aromaticity index of various CTPs were calculated by Eq. (1) reported by Guillén and co-workers [18].

$$
\mathrm{I}_{\text {aromaticity }}=\mathrm{Abs}_{3050} /\left(\mathrm{Abs}_{3050}+\mathrm{Abs}_{2920}\right)
$$

High aromaticity is a well known characteristic of CTP as compared with petroleum pitch [21]. During the various treatments, the aromaticity index increased from 0.76 of raw CTP to $0.88-0.90$.

\subsubsection{Optical properties}

Meso-phase formation was observed by polarized optical microscopy. The optical textures of differently treated CTPs are shown in Fig. 3. The thermally treated (CTP-T) developed many meso-phase particles due to the high reaction temperature of $380^{\circ} \mathrm{C}-400^{\circ} \mathrm{C}$. The sizes of the meso-phase was less than $50 \mu \mathrm{m}$ and the meso-phase particles did not grow forming a large size of lamellar. This texture makes the spinning process difficult. By applying both acid treatments before the thermal treatment, the meso-phase formation was suppressed due to $100^{\circ} \mathrm{C}$ lower reaction temperature. Thus, CTP-S, CTP-N, and CTP-NE were $100 \%$ isotropic precursor pitches. Their catalytic effect on the poly-condensation reaction enabled this low temperature reaction process, and thereby prevented the generation of mesophase [5].

\subsubsection{Distribution of molecular weight and carbon yield}

Although we tried nuclear magnetic resonance (NMR) obtain molecular weight distribution curve, the appropriate NMR solvent for CTP could not be found. The solubility of acetone was very low and peaks of deuterochloroform overlapped with the peaks of CTP. Thus, TGA was conducted to predict the

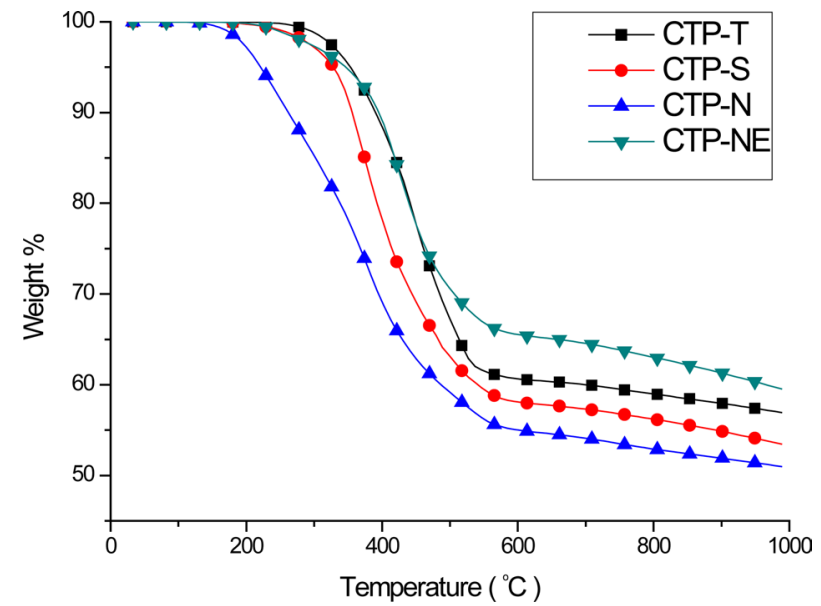

Fig. 4. Thermogravimetric analysis curves of thermal and acid treated pitches having softening point around $220^{\circ} \mathrm{C}$. CTP: coal tar pitch.

distribution of molecular weight, as shown in Fig. 4. The low molecular weight portion of CTP was released in the form of a gas mixture at lower temperatures of $200^{\circ} \mathrm{C}-400^{\circ} \mathrm{C}$. The gradual decrease in weight over a long range of temperature increase indicates that the distribution of molecular weight is broad. For the CTP-N sample, the weight decline started earlier than for the other samples, at around $200^{\circ} \mathrm{C}$, and continued until $600^{\circ} \mathrm{C}$. This broad distribution of molecular weight likely occurred due to the inhomogeneity of the nitric acid and CTP mixture during the poly-condensation reaction. In order to improve the homogeneity, the CTP was mixed with an amphiphilic ethanol solvent before the consecutive nitric acid and thermal treatments. With ethanol addition, the starting temperature of decline increased to $300^{\circ} \mathrm{C}$ and continued until about $550^{\circ} \mathrm{C}$, indicating a narrower distribution of molecular weight. The narrower distribution of molecular weight means that the precursor has homogeneous properties. We can also infer the carbon yield form precursor pitch to carbon fiber by the TGA curves. When the modified CTPs were heated to $1000^{\circ} \mathrm{C}$ in a nitrogen flow, the CTP-N sample had the lowest carbon yield at $51 \%$, and the carbon yields of the other CTPs were $53 \%, 57 \%$, and $60 \%$ for CTP-S, CTP-T, and CTP-NE, respectively. Thus, a precursor pitch with a narrow distribution of molecular weight and a high carbon yield could be obtained by the ethanol and nitric acid treatment.

\subsubsection{Viscosities of modified CTP}

The viscosities of two types of spinnable pitch precursors are represented as a function of temperature in Fig. 5. CTP-AB and CTP-NE showed a similar viscosity of over 700 $000 \mathrm{cP}$ at their $\mathrm{SP}$ and the viscosities decreased sharply between $\mathrm{SP}$ and $\mathrm{SP}+15^{\circ} \mathrm{C}$. In the case of $\mathrm{CTP}-\mathrm{AB}$, its viscosity declined to around $100000 \mathrm{cP}$ at $\mathrm{SP}+30^{\circ} \mathrm{C}$. However, that of CTP-NE: SP236 did not change substantially from $\mathrm{SP}+20^{\circ} \mathrm{C}$ to $\mathrm{SP}+70^{\circ} \mathrm{C}$ and then decreased again, reaching $100000 \mathrm{cP}$ at $\mathrm{SP}+85^{\circ} \mathrm{C}$. It was demonstrated that the catalytic effects of nitric acid accelerated the poly condensation and crosslinking, showing an irregular viscosity curve for CTP-NE. The suitable temperature for pitch spinning is known as the point where the viscosity is about $80000 \mathrm{cP}$ at 3 bar. Thus, 


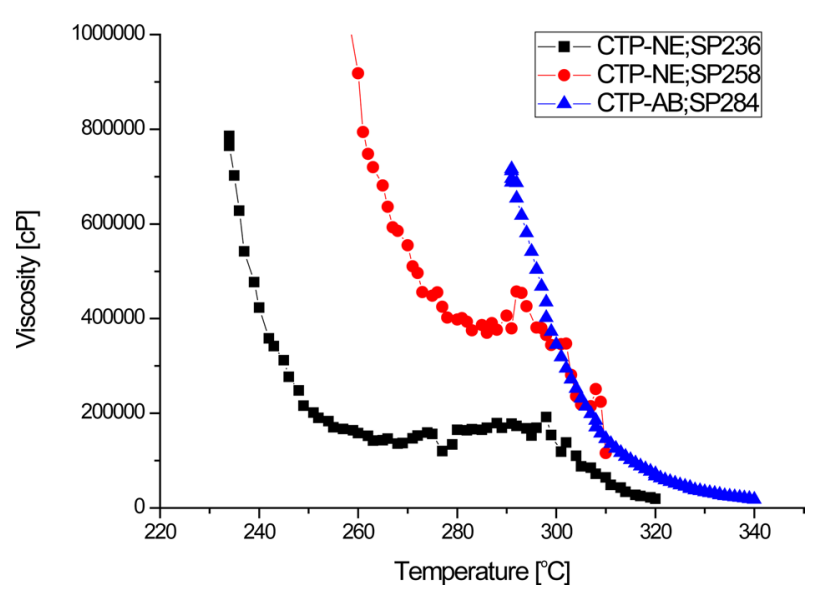

Fig. 5. Viscosities of modified coal tar pitches (CTPs) depending on temperature. the spinning temperature of $\mathrm{CTP}-\mathrm{AB}$ was chosen as $314^{\circ} \mathrm{C}$ $\left(\mathrm{SP}+30^{\circ} \mathrm{C}\right)$. However, since $\mathrm{CTP}-\mathrm{NE}$ maintained a viscosity interval, this point was not suitable for spinning because of oxidation of the pitch and gas emission at high temperature. CTP-NE was spun at $\mathrm{SP}+35^{\circ} \mathrm{C}$ and higher pressure of more than 8 bar was required due to the high viscosity.

\subsection{Properties of carbon fibers}

The SEM images of carbon fibers for CTP-AB and CTP-NE are shown Fig. 6. The average diameters (ADs) of as-spun fibers with CTP-NE and AB were $21.3 \mu \mathrm{m}$ and $14.9 \mu \mathrm{m}$, respectively. This difference was caused by the differences in viscosity and spinning pressure. After stabilization, the AD of CTP-NE slightly increased to $21.8 \mu \mathrm{m}$ because of oxygen permeation. On the other hand, that of CTP-AB decreased to $13.0 \mu \mathrm{m}$. Since $\mathrm{CTP}-\mathrm{AB}$ underwent sufficient oxygen combination during air

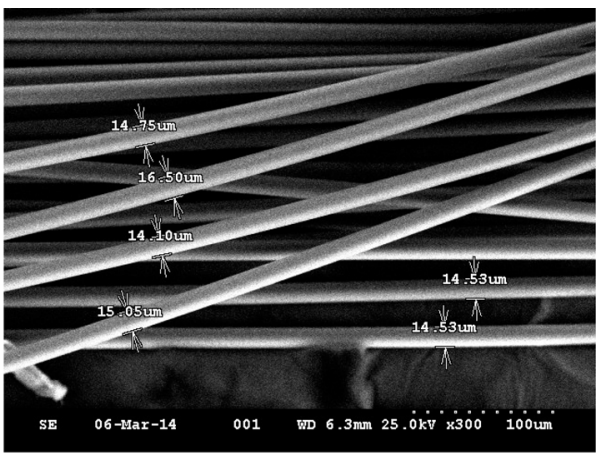

(a) As-spun fibers-CTP-NE: $\mathrm{AD}=21.3 \pm 0.7 \mu \mathrm{m}$

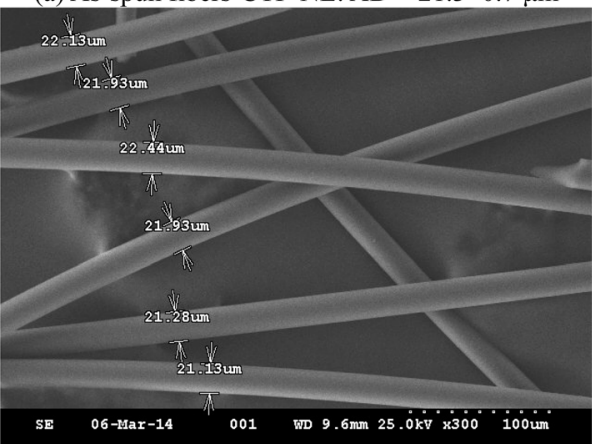

(c) Stabilized fibers-CTP-NE: AD $=21.8 \pm 0.7 \mu \mathrm{m}$

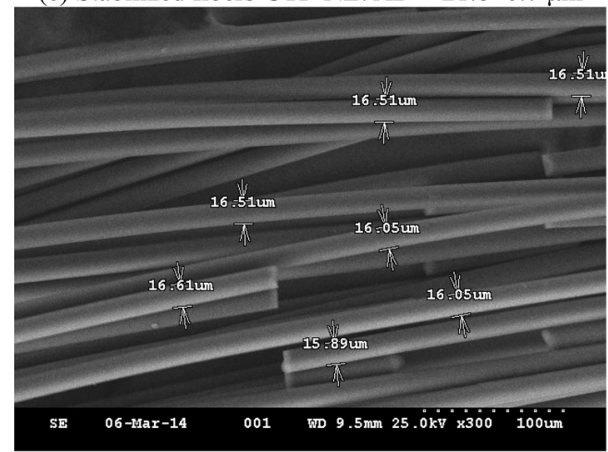

(e) Carbonized fibers-CTP-NE: $\mathrm{AD}=16.3 \pm 0.3 \mu \mathrm{m}$ (b) As-spun fibers-CTP-AB: AD $=14.9 \pm 1.6 \mu \mathrm{m}$

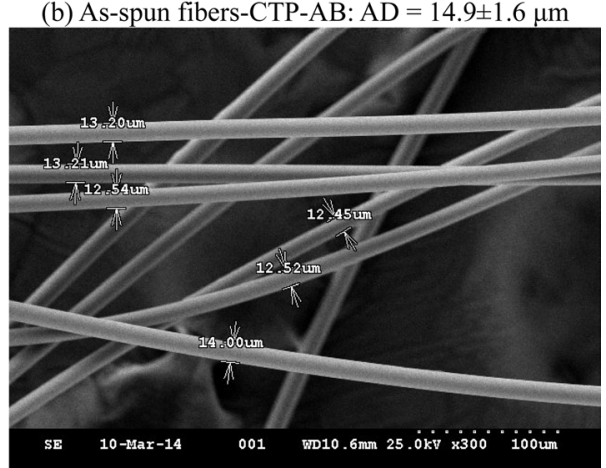

(d) Stabilized fibers-CTP-AB: AD = 13.0 $\pm 1.0 \mu \mathrm{m}$

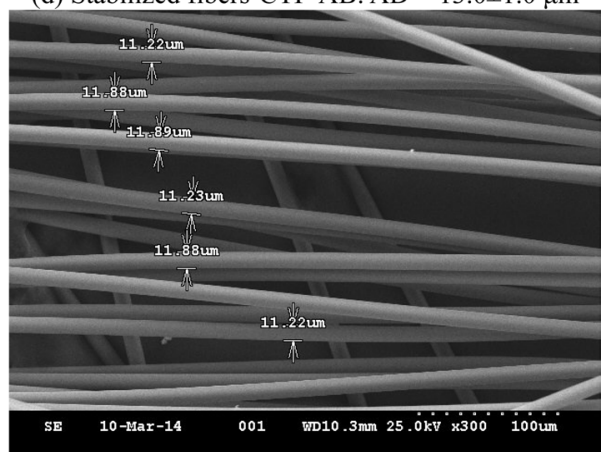

(f) Carbonized fibers-CTP-AB: $\mathrm{AD}=11.6 \pm 0.4 \mu \mathrm{m}$

Fig. 6. Scanning electron microscope images of various fibers with treatment conditions. CTP: coal tar pitch, AD: average diameter. 
blowing treatment, the diameter decreased due to the gas emission during stabilization. During the carbonization process, their diameters decreased to 16.3 and $11.6 \mu \mathrm{m}$, respectively. The light gas of carbon fiber was eliminated by heating. The as-spun and stabilized fibers with CTP-AB appeared glossier in SEM images than those with CTP-NE and this difference was less pronounced after the carbonization.

\section{Conclusions}

In order to prepare a spinnable precursor pitch with proper properties for carbon fiber using isotropic CTP, we investigated various treatments on CTP such as thermal treatment only and sulfuric acid treatment or nitric acid treatment before thermal treatment. The thermal treated CTP required a relatively high reaction temperature of more than $380^{\circ} \mathrm{C}$ to prepare a precursor pitch with a high SP for carbon fiber and it had many mesophase particles that were created during application of high reaction temperature. When nitric acid or sulfuric acid treatment was conducted before the thermal treatment, a precursor pitch with a proper SP range could be obtained at reaction temperatures of $280^{\circ} \mathrm{C}-300^{\circ} \mathrm{C}$. With the acid treatments, the yield and SP of the precursor pitch increased dramatically and the formation of a meso-phase was suppressed due to the lower reaction temperatures. However, in the case of the sulfuric acid treatment, it is too difficult to control optimal reaction conditions due to the sensitive temperature dependence. The nitric acid treated CTP also had problems of a broad distribution of molecular weight and low fluidity at spinning temperature due to the inhomogeneity of the nitric acid and CTP mixture during the poly-condensation reaction. By adopting the nitric acid treatment with ethanol, CTP achieved more homogeneous properties such as a short range of viscosity and a narrow distribution of molecular weight. The CTP with ethanol, nitric acid, and thermal treatments could be spun continuously, and their spinning and carbon fiber properties were compared to those of the air blowing and thermal treated CTP.

\section{Acknowledgements}

This research was financially supported by the "Carbon Valley Construction Program" through the Ministry of Trade, Industry \& Energy (MOTIE) and Korea Institute for Advancement of Technology (KIAT).

\section{References}

[1] Wazir AH, Kakakhel L. Preparation and characterization of pitchbased carbon fibers. New Carbon Mater, 24, 83 (2009). http:// dx.doi.org/10.1016/S1872-5805(08)60039-6.

[2] Yu B, Wang C, Chen M, Zheng J, Qi J. Two-step chemical conversion of coal tar pitch to isotropic spinnable pitch. Fuel Process Technol, 104, 155 (2012). http://dx.doi.org/10.1016/j.fuproc. 2012.05.007.

[3] Yoon SH, Korai Y, Mochida I. Assessment and optimization of the stabilization process of mesophase pitch fibers by thermal analyses. Carbon, 32, 281 (1994). http://dx.doi.org/10.1016/00086223(94)90191-0.

[4] Menéndez R, Gray EM, Marsh H, Pysz RW, Heintz EA. The influence of primary quinoline insolubles on the development of secondary quinoline insolubles in coal tar pitches. Carbon, 29, 107 (1991). http://dx.doi.org/10.1016/0008-6223(91)90101-N.

[5] Petrova B, Budinova T, Petrov N, Yardim MF, Ekinci E, Razvigorova M. Effect of different oxidation treatments on the chemical structure and properties of commercial coal tar pitch. Carbon, 43, 261 (2005). http://dx.doi.org/10.1016/j.carbon.2004.09.006.

[6] Blanco C, Santamaría R, Bermejo J, Menéndez R. A comparative study of air-blown and thermally treated coal-tar pitches. Carbon, 38, 517 (2000). http://dx.doi.org/10.1016/S0008-6223(99)00131-1.

[7] Zeng SM, Maeda T, Tokumitsu K, Mondori J, Mochida I. Preparation of isotropic pitch precursors for general purpose carbon fibers (GPCF) by air blowing: II. Air blowing of coal tar, hydrogenated coal tar, and petroleum pitches. Carbon, 31, 413 (1993). http:// dx.doi.org/10.1016/0008-6223(93)90128-W.

[8] Ko HJ, Park CU, Cho HH, Yoo MJ, Kim MS, Lim YS. Preparation of coal tar pitch as carbon fibers precursor form coal tar. Korean J Mater Res, 23, 276 (2013). http://dx.doi.org/10.3740/ MRSK.2013.23.5.276.

[9] Domínguez A, Blanco C, Santamaría R, Granda M, Blanco CG, Menéndez R. Monitoring coal-tar pitch composition changes during air-blowing by gas chromatography. J Chromatogr A, 1026, 231 (2004).

[10] Lewis IC. Thermal polymerization of aromatic hydrocarbons. Carbon, 18, 191 (1980). http://dx.doi.org/10.1016/0008-6223(80) 90060-3

[11] Cheng X, Zha Q, Li X, Yang X. Modified characteristics of mesophase pitch prepared from coal tar pitch by adding waste polystyrene. Fuel Process Technol, 89, 1436 (2008). http://dx.doi.org/ 10.1016/j.fuproc.2008.07.003.

[12] Li T, Liu X, Wang C, Wang H. Structural characteristics of mesophase spheres prepared from coal tar pitch modified by phenolic resin. Chin J Chem Eng, 14, 660 (2006). http://dx.doi.org/10.1016/ S1004-9541(06)60131-6.

[13] Lin Q, Li T, Zheng C, Zhao Y, Song S. Carbonization behavior of coal-tar pitch modified with divinylbenzene and optical texture of resultant semi-cokes. J Anal Appl Pyrolysis, 71, 817 (2004). http:// dx.doi.org/10.1016/j.jaap.2003.10.009.

[14] Machnikowski J, Kaczmarska H, Gerus-Piasecka I, Díez MA, Alvarez R, García R. Structural modification of coal-tar pitch fractions during mild oxidation: relevance to carbonization behavior. Carbon, 40, 1937 (2002). http://dx.doi.org/10.1016/S00086223(02)00029-5.

[15] Panaitescu C, Predeanu G. Microstructural characteristics of toluene and quinoline-insolubles from coal-tar pitch and their cokes. Int J Coal Geol, 71, 448 (2007). http://dx.doi.org/10.1016/j. coal.2006.11.003.

[16] Granda M, Casal E, Bermejo J, Menéndez R. The influence of primary QI on the oxidation behaviour of pitch-based $\mathrm{C} / \mathrm{C}$ composites. Carbon, 39, 483 (2001). http://dx.doi.org/10.1016/S00086223(01)00006-9.

[17] Marsh H, Latham CS, Gray EM. The structure and behaviour of QI material in pitch. Carbon, 23, 555 (1985). http://dx.doi.org/ 10.1016/0008-6223(85)90092-2.

[18] Guillén MD, Iglesias MJ, Domínguez A, Blanco CG. Fourier transform infrared study of coal tar pitches. Fuel, 74, 1595 (1995). 
http://dx.doi.org/10.1016/0016-2361(95)00139-V.

[19] Akezuma M, Okuzawa K, Esumi K, Meguro K, Honda H. Physicochemical properties of quinoline-soluble and quinolineinsoluble mesophases. Carbon, 25, 517 (1987). http://dx.doi.org/ 10.1016/0008-6223(87)90192-8.

[20] Ōtani S. Mechanism of the carbonization of MP carbon fiber at the low temperature range. Carbon, 5, 219 (1967). http://dx.doi.org/ 10.1016/0008-6223(67)90003-6.

[21] Gray RJ, Krupinski KC. Pitch production: supply, coking, optical microscopy and application. In: Marsh H, Heintz EA, RodríguezReinoso F, eds. Introduction to Carbon Technologies, Universidad de Alicante, Alicante, Spain, 329 (1997). 